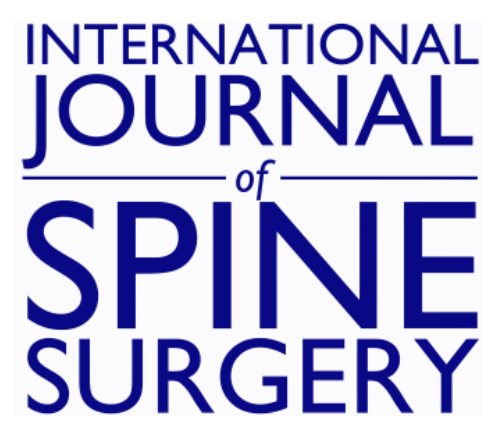

\title{
Biomechanical Behavior of a New Nucleus Prosthesis Made of Knitted Titanium Filaments
}

Annette Kettler, Hans-Peter Kaps, Bodo Haegele and Hans-Joachim Wilke

Int J Spine Surg 2007, 1 (4) 125-130

doi: https://doi.org/10.1016/SASJ-2007-0106-RR

http://ijssurgery.com/content/1/4/125

This information is current as of April 26, 2023.

Email Alerts Receive free email-alerts when new articles cite this article. Sign up at:

http://ijssurgery.com/alerts

The International Journal of Spine Surgery

2397 Waterbury Circle, Suite 1,

Aurora, IL 60504, Phone: +1-630-375-1432 


\title{
Biomechanical Behavior of a New Nucleus Prosthesis Made of Knitted Titanium Filaments
}

Annette Kettler, MD, Hans-Peter Kaps, PhD, Bodo Haegele, MD, and Hans-Joachim Wilke, PhD

\begin{abstract}
Background

One of the greatest challenges in the development of a nucleus prosthesis is to minimize the risk of implant expulsion. At the same time, the physiological flexibility, compressive behavior, and height of the disc should be restored. In this biomechanical in vitro study we investigated the ability of a new nucleus prosthesis made of knitted titanium filaments to meet these challenges.
\end{abstract}

\section{Methods}

Flexibility, axial deformation, and height of six bovine lumbar spine segments were measured in the intact condition, after implantation of the new prosthesis, and during and after complex cyclic loading $(100,000$ cycles). For this purpose, six new prostheses preformed according to the shape of the bovine nucleus pulposus were manufactured. Flexibility was tested in the three main planes under pure moment loads of 7.5 Nm. Axial deformation was measured under application of an axial force of $1000 \mathrm{~N}$. Radiographs taken before and after cyclic testing were used to assess implant migration and expulsion.

\begin{abstract}
Results
In lateral bending, the intact range of motion (RoM) could almost be restored after implantation. However, in axial rotation, the RoM increased slightly with the implant. This was also the case in extension, with an increase from $-2.9^{\circ}$ to $-6.4^{\circ}$, whereas in flexion, RoM decreased from $4.3^{\circ}$ to $3.2^{\circ}$. In all loading planes, cyclic loading caused the RoM to increase asymptotically by $0.1^{\circ}$ to $1.8^{\circ}$. The axial deformation of the specimens was nearly equivalent in all tested states, as was their height. Cyclic loading did not cause implant expulsion.

Conclusions

In this feasibility study, the new knitted nucleus prosthesis showed promising results in segmental flexibility, axial deformability, height, and implant expulsion. However, further study is needed for other factors, such as wear and fatigue behavior.
\end{abstract}

Key Words Lumbar spine, intervertebral disc, nucleus prosthesis, titanium, biomechanics. SAS Journal. Autumn 2007; 1:125-130. DOI: SASJ-2007-0106-RR

\section{INTRODUCTION}

Nonfusion implants have become increasingly popular in the treatment of painful intervertebral disc or facet joint disorders. Some of these implants, such as intervertebral disc prostheses ${ }^{1}$ and dynamic posterior stabilization systems, ${ }^{2}$ are already used in clinical practice, whereas others, including posterior element replacements ${ }^{3}$ or nucleus prostheses, are still under investigation. Various types of nucleus prostheses have been and are still being developed. ${ }^{4}$ They are all implanted to replace the nucleus while leaving the annulus fibrosus as intact as possible. Their mechanical characteristics mostly mimic those of the healthy nucleus in order to restore the natural mechanical behavior of the disc. The most extensively studied nucleus implant is the Prosthetic Disc Nucleus (PDN; Raymedica, Minneapolis, Minnesota). The PDN is made of a water-absorbing hydrogel core encased in a polyethylene jacket. Whereas some early clinical trials for the PDN showed promising results, ${ }^{5}$ in other studies migration rates up to $35 \%$ were reported. ${ }^{6}$ Although further development of the implant design reduced its migration rate, an element of risk remains. Similar problems have been reported for nucleus prostheses made of polyvinyl alcohol (PV) or collagen. ${ }^{7.8}$

Ideally, a nucleus implant should not migrate or be expulsed. In addition, the implant should restore the physiological flexibility, compressive behavior, and height of the treated disc. Based on these requirements, Buck TSP(Bondorf, Germany) has developed a preformed nucleus prosthesis made of knitted titanium filaments (Figure 1). Its rough surface is meant to prevent migration, and its deformable architecture is thought to restore normal flexibility. In this biomechanical in vitro study we investigated the ability of the new prosthesis to restore and maintain flexibility, axial deformability, and height of the treated segment and to resist migration and expulsion under complex cyclic loading. 


\section{SASJournal}

Figure 1

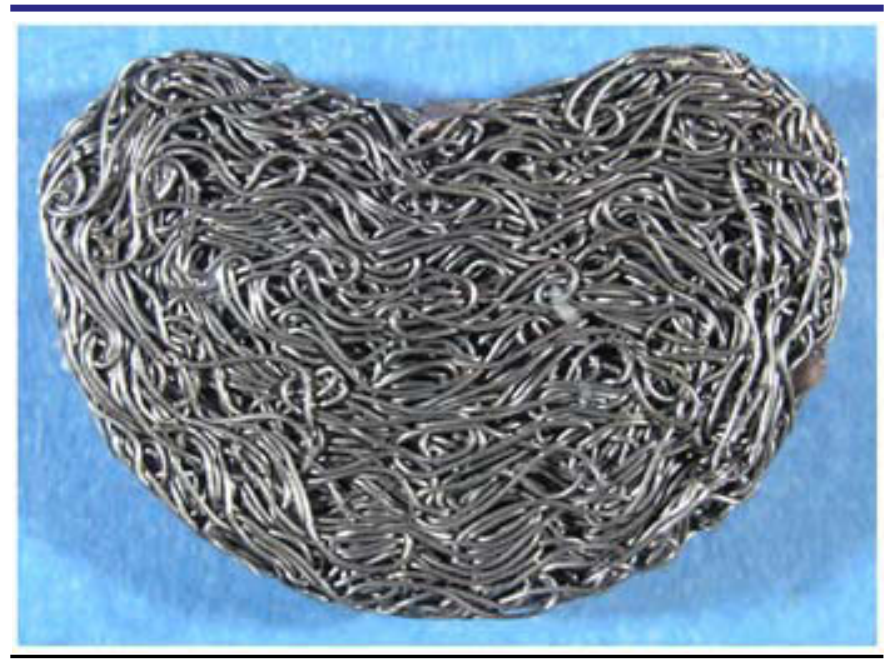

New nucleus prosthesis made of knitted titanium filaments.

\section{MATERIALS AND METHODS}

\section{Specimens}

Six bovine lumbar spine segments (three L2 -3 and three L4-5) were freshly dissected and frozen at $-20^{\circ} \mathrm{C}$ for storage. Several hours before testing, the specimens were thawed, and all soft tissue was removed, taking care to preserve the intervertebral disc, ligaments, and joint capsules. The upper part of the cranial vertebra and the lower part of the caudal vertebra were embedded in polymethylmetacrylate to allow fixation in the testing devices.

\section{Implant}

For this study, six knitted prostheses were manufactured, one for each of the six specimens. The implants were preformed according to the shape of the bovine nucleus pulposus. The new prostheses were implanted into the segments from an anterior approach after fenestration of the annulus. During fenestration two annulus flaps were created extending from the midline toward the outer thirds of the disc (Figure 2). The nucleus pulposus and cartilaginous endplates were removed, and, after implantation, the annulus flaps were sutured. Because this was a feasibility study, specially designed instruments for implantation were not yet available. For the same reason, implantation was not carried out under fluoroscopic control.

\section{Flexibility Tests}

The flexibility of the segments was assessed in a spine tester ${ }^{9}$ under pure moment loads of $7.5 \mathrm{Nm}$ in flexion/extension, lateral bending, and axial rotation. Three loading cycles were applied in each of these three loading planes. During loading, the specimens were not constrained in their movement within the five uncontrolled degrees of freedom. The three-dimensional flexibility of the segments was continuously recorded using rotary variable displacement transducers (Novotechnik, Ostfildern, Germany, resolution $0.1^{\circ}$ ) integrated into the spine tester. Based on the curves of the third loading cycle, range

\section{Figure 2}

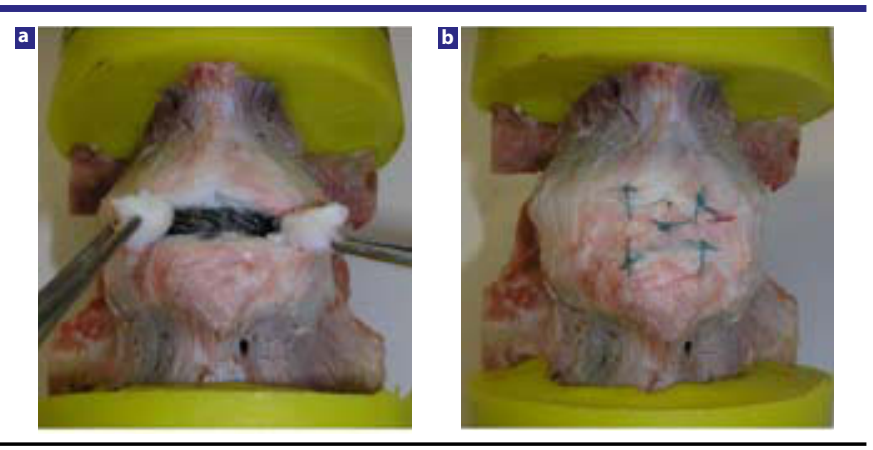

(a) The new nucleus prosthesis was implanted after anterior fenestration of the annulus fibrosus. (b) Two lateral annulus flaps were created and sutured after implantation.

of motion (RoM) was determined as the rotation at maximum load. Additionally, these curves were used to determine the sagittal tilt caused by implantation and its changes during cyclic loading.

\section{Axial Deformability and Specimen Height}

We measured the axial deformability and the height of the specimens in a materials testing machine (Instron 8871; Instron Wolpert GmbH, Darmstadt, Germany). To measure the axial deformability, we applied an axial force through the center of the specimens. This force quasi-statically increased from 100 to $1000 \mathrm{~N}$. We chose an axial force of $1000 \mathrm{~N}$ to mimic the axial preload acting on the human lumbar spine during normal everyday activities. ${ }^{10}$ During measurement, we recorded the resulting deformation. For the height measurements, we applied an axial preload of $100 \mathrm{~N}$. During application of this load, we recorded the position of the piston of the materials testing machine. This value was set to zero for the intact condition.

\section{Test Protocol and Cyclic Loading}

All tests (flexibility, axial deformation, and height) were carried out in the intact condition, directly after implantation and after $20,000,40,000,60,000$, and 100,000 complex loading cycles. An axial force sinusoidally ranging between 100 and $600 \mathrm{~N}$ was applied at a frequency of $5 \mathrm{~Hz}$. The acting line of this force passed the specimen's center (defined as the posterior edge of the intervertebral disc in the midsagittal plane) via a lever arm of $30 \mathrm{~mm}$. Thus, the force additionally created a bending moment ranging between 3 and $18 \mathrm{Nm}$. During loading, the specimens were mounted on a motor-driven platform. On this platform the specimens continuously rotated around their longitudinal axis at a velocity of $360 \%$ min. This rotation caused the plane in which the bending moment was applied to rotate around the specimens continuously. Overall, 100,000 loading cycles were applied in this manner.

\section{Evaluation of Implant Migration and Expulsion}

We assessed the migration of the implant using plain posteroanterior and lateral radiographs taken before and after cyclic 
loading. We matched the pre-test and post-test radiographs according to the bony structures and compared the two sets of images, and we subsequently compared the implant position before and after testing. Because we did not account for image magnification, the magnitude of the migration reported in our results (see following text) should be considered a trend. After cyclic testing, the specimens were cut in the transverse plane through the disc to allow inspection of the implant, implant position, and implant bed macroscopically.

\section{Statistics}

We used the Wilcoxon signed rank test for statistical evaluation of the data. Because this study was meant to give an overview of implant performance with respect to several biomechanical characteristics, the $P$ values were not corrected for multiple comparisons. Therefore the data show a trend but should not be considered as statistical evidence.

\section{RESULTS}

\section{Flexibility}

In the flexibility tests, the RoM in flexion decreased from $4.3^{\circ}$ (median value) in the intact condition to $3.2^{\circ}$ after implantation, whereas in extension it increased from $-2.9^{\circ}$ to $-6.4^{\circ}(P<0.05)$ (Figure 3). Thus, after implantation, the RoM was more than twice as high in extension than in flexion. This asymmetry can be explained by the lordotic tilt (median, $-1.7^{\circ}$ ) caused by implantation. In contrast, in lateral bending, the RoM of the intact specimens came close to being restored (Figure 4), whereas in axial rotation the increase was small, at $0.7^{\circ}$ (median) for axial rotation to the left and $-0.8^{\circ}$ (median) for axial rotation to the right (Figure 5). In all loading planes cyclic loading caused the RoM to increase. This increase was smaller in flexion $\left(0.4^{\circ}\right)$ and axial rotation $\left(0.1^{\circ}\right.$ and $\left.-0.5^{\circ}\right)$ than in extension $\left(-1.4^{\circ}\right)$ and lateral bending $\left(1.8^{\circ}\right.$ and $\left.-1.1^{\circ}\right)$ but always had an asymptotic progression.

\section{Figure 3}

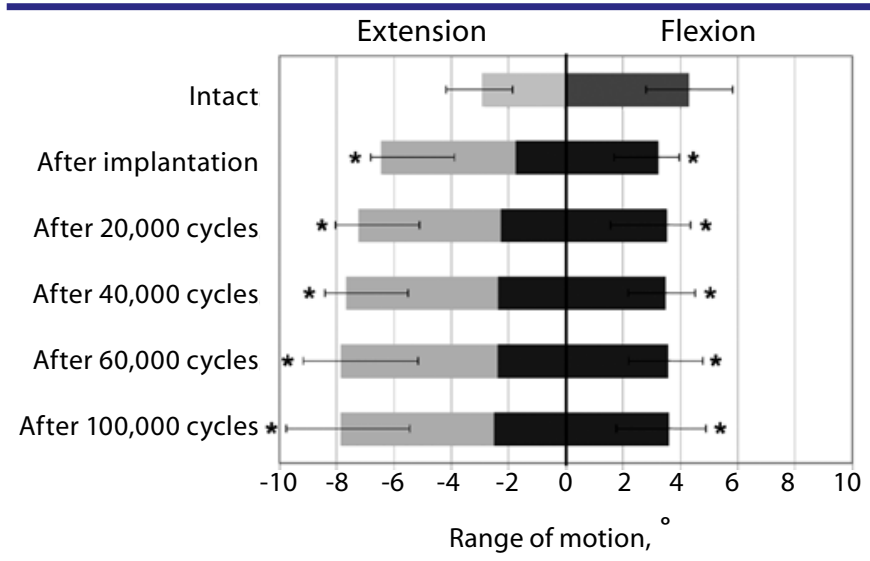

Range of motion in flexion and extension in the intact condition, after implantation, and during and after cyclic loading. The border between the light and dark parts of each bar represents the median lordotic tilt caused by implantation and cyclic loading. Median with range is shown.

* $P<0.05$ compared to the intact condition.

\section{Figure 4}

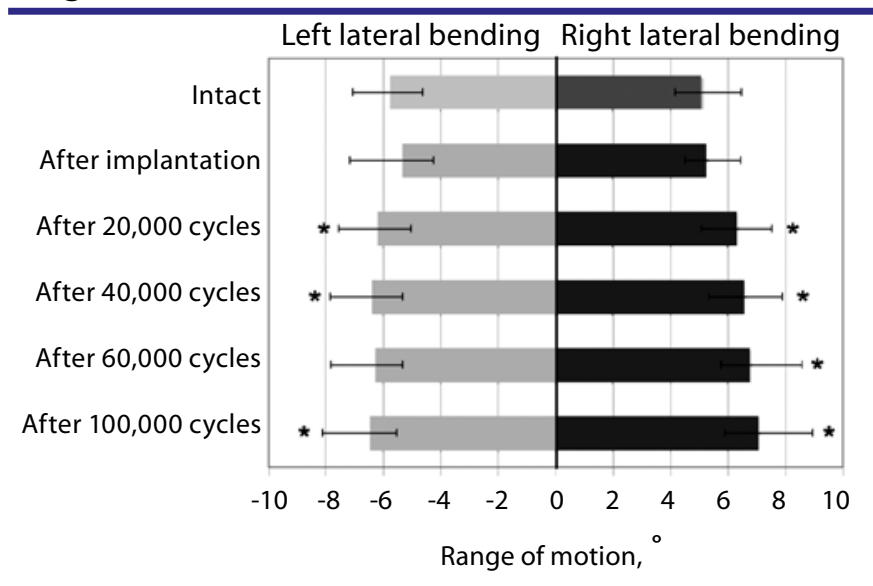

Range of motion in flexion and extension in the intact condition, after implantation, and during and after cyclic loading. The border between the light and dark parts of each bar represents the median lordotic tilt caused by implantation and cyclic loading. Median with range is shown. ${ }^{*} P<0.05$ compared to the intact condition.

Figure 5

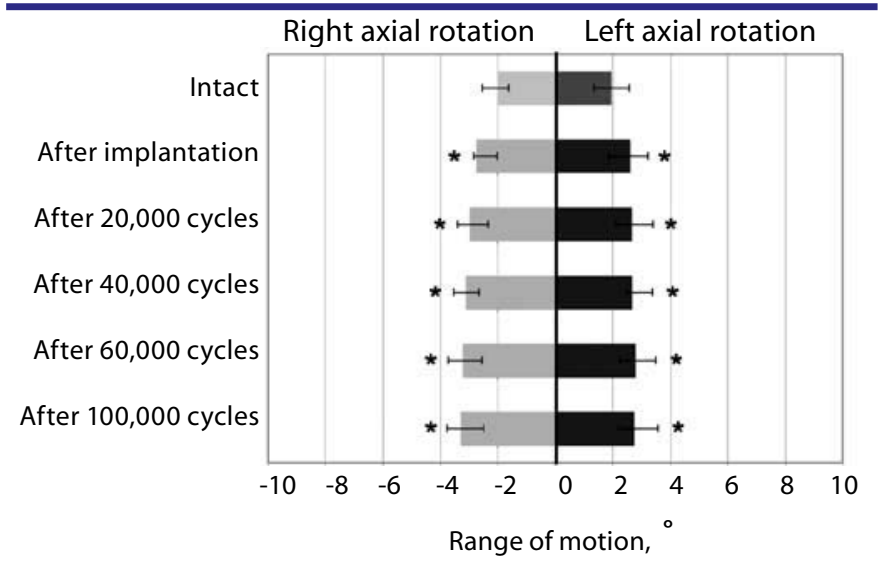

Range of motion in axial rotation in axial rotation in the intact condition, after implantation, and during and after cyclic loading. Median with range is shown.

${ }^{*} P<0.05$ compared to the intact condition.

\section{Axial Deformability}

The axial deformation of the specimens was almost the same in all tested states (Table 1). In the intact and implanted states it was $-0.4 \mathrm{~mm}$ (median), and during and after cyclic loading it was $-0.3 \mathrm{~mm}(P>0.05)$.

\section{Specimen Height}

The height of the specimens did not change with implantation (Table 2). Also, cyclic loading only caused a small height loss of $0.7 \mathrm{~mm}$ (median, $P>0.05)$.

\section{Implant Migration and Expulsion}

Implant expulsion was not observed in any of the six specimens. Also, significant loosening of the annulus suture was not observed. However, on the radiographs a migration 
Table 1

\begin{tabular}{lccc}
\hline \multicolumn{4}{l}{ Axial Deformation Under an Axial Load Increasing From 100 to $1000 \mathrm{~N}$} \\
\hline Axial deformation, mm & Median & Minimum & Maximum \\
\hline Intact & -0.4 & -0.5 & -0.2 \\
After implantation & -0.4 & -0.4 & -0.3 \\
After 20,000 cycles & -0.3 & -0.4 & -0.3 \\
After 40,000 cycles & -0.3 & -0.4 & -0.3 \\
After 60,000 cycles & -0.3 & -0.4 & -0.3 \\
After 100,000 cycles & -0.3 & -0.4 & -0.3 \\
\hline
\end{tabular}

Table 2

\begin{tabular}{|c|c|c|c|}
\hline Height change, $\mathrm{mm}$ & Median & Minimum & Maximum \\
\hline Intact & 0 & 0 & 0 \\
\hline After implantation & 0 & 1.2 & -0.3 \\
\hline After 20,000 cycles & -0.5 & 1.3 & -1.3 \\
\hline After 40,000 cycles & -0.6 & 1 & -1.4 \\
\hline After 60,000 cycles & -0.6 & 1.1 & -1.4 \\
\hline After 100,000 cycles & -0.7 & 0.9 & -1.5 \\
\hline
\end{tabular}

of the implants in the anterior direction was detectable. This migration was no more than $1 \mathrm{~mm}$ in four specimens (Figure 6), but about $2 \mathrm{~mm}$ and $3 \mathrm{~mm}$ of migration was observed in the remaining two specimens. Macroscopic inspection confirmed these findings. In these two specimens, the cavity created for implantation was larger than the implant itself, which probably allowed the implant to migrate.

\section{Figure 6}

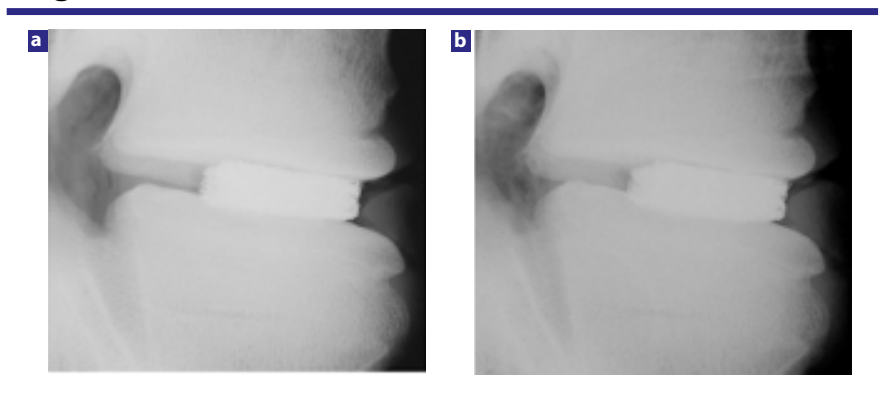

(a) Lateral radiograph of one of the six specimens directly after implantation. (b) After complex cyclic loading the position of the prosthesis is almost the same as before, yet a slight migration in anterior direction is observable .

\section{DISCUSSION}

In this biomechanical in vitro study a new nucleus prosthesis made of knitted titanium filaments was tested for its ability to restore and maintain flexibility, axial deformability, and height of the healthy segment and to resist migration and expulsion.

\section{Flexibility}

In the flexibility tests, RoM could be restored in lateral bending but was increased in axial rotation. However, this increase was less than $0.5^{\circ}$. Likewise, in flexion/extension, RoM could not be restored completely: in flexion a decrease was recorded, while in extension an increase was found. In this loading plane the performance of the new prosthesis could probably be improved if the prosthesis were implanted more toward the center of the disc. This position would probably be possible if special instruments for implantation were available and if implantation was carried out under fluoroscopic control. For comparison, a good restoration of the three-dimensional flexibility in all three loading planes was shown for the PDN. ${ }^{11}$ In the present study, cyclic loading caused the RoM to increase. This increase had an asymptotic progression, which almost reached its maximum after 100,000 loading cycles. Therefore, only a small amount of destabilization can be expected beyond 100,000 cycles.

\section{Axial Deformability}

In the axial compression tests, the segments maintained their natural deformability. For comparison, compressive tests have also been reported for the PVA and polyvinyl pyrrolidone (PVP) implant. ${ }^{12}$ In this study, as in ours, the natural axial stiffness of the segments came close to being restored.

\section{Segment Height}

In addition to the restoration in axial deformability, the segment height was also restored with the new knitted implant. Again, similar results have been reported for other nucleus implants, such as the spiral implant (Newcleus, Zimmer spine, Warsaw, Indiana $)^{4,13}$ and the PDN. ${ }^{11}$ This parameter is clinically relevant because insufficient height restoration or height loss can lead to a narrowing of the neuroforamen and to nerve root compression.

\section{Implant Migration and Expulsion}

We observed no expulsion in any of the six specimens even though the annulus fenestration covered almost two thirds of the anterior aspect of the disc and stringent loading conditions were applied. This observation applies to both axial compression and bending. The compressive force was meant to mimic the axial preload, which is assumed to act on the human lumbar spine in a lying-down $(100 \mathrm{~N})$ or standing $(600 \mathrm{~N})$ posture. ${ }^{10}$ Because this force was applied eccentrically via a lever arm of $30 \mathrm{~mm}$, the specimens were also loaded with a bending moment of up to 18 $\mathrm{Nm}$ - more than twice the bending moment recommended for spinal implant testing, which is $7.5 \mathrm{Nm} .{ }^{14}$ Thus, we suspect that a poorly anchored implant might indeed have migrated under these loading conditions. Other nucleus implants were reported to migrate or to be extruded even during standard flexibility tests, including a nucleus implant made of collagen-type-I. ${ }^{8}$ However, the annulus defect created to implant this collagen implant was not sutured. Similar results were reported in an animal study with baboons, in which expulsion of PVA implants occurred in up to $33 \%$ of cases. $^{7}$ Further investigation is needed to show whether the suture used to close the annulus defect created in 
the present study remains effective over a longer period of time and whether some kind of annular healing is possible.

Although expulsion could not be provoked in our study, some implant migration was detectable. Whether this migration would eventually lead to expulsion is difficult to answer because the 20-hour maximum recommended for preparation and testing means that 100,000 cycles could not be completed. ${ }^{14}$ However, macroscopic inspection showed that implant migration mainly occurred within the cavity created by the surgeon: in cases where the cavity was larger than the implant, migration was observed, whereas in cases where cavity and implant were similar in size, migration was minimal. Thus, further loading would probably not have led to further implant migration or expulsion. Nevertheless, care should be taken not to create a cavity larger than the implant. Besides the size of the cavity, endplate preparation may also play a role in implant anchorage. In the present study the cartilaginous endplates were removed. Whether implant anchorage would be significantly affected if the cartilaginous endplates were left intact still needs to be investigated.

Migration was always directed toward the anterior border of the disc. This can be explained, first, by the anterior approach and, second, by the position of the implants, which tended to be anterior to the center of the disc. As mentioned previously, this position can probably be improved if special instruments are used for implantation and if implantation is carried out under fluoroscopic control.

\section{Cyclic Loading}

To our knowledge, so far, similar cyclic loading tests have not been carried out with other nucleus prostheses. In contrast, the material properties and fatigue behavior of some of these implants is well known. PVA and PVP, used in the Aquarelle implant (Stryker Spine, Allendale, New Jersey), as well as a fiber-reinforced material used in the NeuDisc (Replication Medical Inc., New Brunswick, New Jersey), successfully passed fatigue tests, and these implants were shown to mimic the mechanical behavior of the nucleus. ${ }^{12,15,16}$ In a preliminary study, the new knitted prosthesis was also tested for fatigue and wear; 1.6 million axial load cycles were applied (100-1000 N) to a cylindrical sample made of the new knitted material, which was placed in a physiological saline solution. The weight of the sample was recorded before and after testing. The difference in weight was only $0.003 \mathrm{~g}$, indicating a probable lack of significant wear. Further wear tests are needed to show whether this is the case for more than 1.6 million loading cycles.

A few broken filaments were detected after testing. Whether the filaments had broken during the manufacturing process or if the breakage occurred during cyclic loading is difficult to ascertain. In either case, the broken filaments would put the patient at further risk. Therefore, care should be taken to check the implants and discard broken filaments before implantation.

\section{Animal Model}

We used bovine specimens because of the limited availability of human specimens and because the lumbar spine of the calf is similar to that of the human with respect to endplate dimensions and flexibility. ${ }^{17,18}$ The calf therefore seemed to be an adequate model to establish a first impression of how the new nucleus implant behaves. However, from a quantitative perspective the results may differ from those expected in the human. The final version of the new implant should therefore be tested using human specimens.

Subsidence tests are not feasible using calf spine specimens due to their relatively high bone mineral density. Therefore, it is not surprising that none of the six specimens showed signs of endplate damage after testing. Yet, even in human specimens the risk of subsidence is assumed to be small because the shape of the new knitted prosthesis adapts to the curvature of the endplates due to its deformable design. Therefore no stress concentrations are expected. Furthermore, in preliminary experiments, the stiffness of the prosthesis was optimized until the physiological axial deformation of a healthy segment could be restored (Table 1).

Overall, the results of this study were promising. The most important advantage of the new knitted nucleus prosthesis seems to be its low migration tendency. In addition, its ability to restore and maintain the physiological axial deformability and the height of the treated segment is almost equivalent to that of other nucleus implants. Only the restoration of the three-dimensional flexibility was not as good as reported for competitive implants. In this case the performance of the new implant can probably be improved if special instruments are used for implantation and if implantation is carried out under fluoroscopic control. One of the next steps would therefore be to develop special instruments for implantation. Further evaluation should additionally focus on such factors as wear and tear and fatigue behavior.

Annette Kettler, MD, Hans-Peter Kaps, PhD, Bodo Haegele, MD, HansJoachim Wilke, PhD

From the Institute for Orthopaedic Research and Biomechanics, University of Ulm, Germany (Kettler, Haegele, and Wilke), and the Department of Orthopaedics, BG Trauma-Centre, Tuebingen, Germany (Kaps).

This work was supported by Buck TSP, Bondorf, Germany.

Address correspondence and reprint requests to Hans-Joachim Wilke, PhD, Institute for Orthopaedic Research and Biomechanics, University of Ulm, Helmholtzstr. 14,D-89081 Ulm Germany (email: hans-joachim.wilke@uni-ulm.de)

This manuscript was submitted on September 19, 2007, and accepted for publication on October 14, 2007. 


\section{REFERENCES}

1. Anderson PA, Rouleau JP. Intervertebral disc arthroplasty. Spine. 2004;29:2779-2786.

2. Grob D, Benini A, Junge A, et al. Clinical experience with the Dynesys semirigid fixation system for the lumbar spine: surgical and patientoriented outcome in 50 cases after an average of 2 years. Spine. 2005;30:324-331.

3. Wilke HJ, Schmidt H, Werner K, et al. Biomechanical evaluation of a new total posterior-element replacement system. Spine. 2006;31:27902796.

4. Di Martino A, Vaccaro AR, Lee JY, et al. Nucleus pulposus replacement: basic science and indications for clinical use. Spine. 2005;30:S16-22.

5. Bertagnoli R, Schonmayr R. Surgical and clinical results with the PDN prosthetic disc-nucleus device. Eur Spine J. 2002;11(Suppl 2): S143-S148.

6. Schonmayr R. The PDN disc nucleus prosthesis: experiences, technical considerations, and clinical results 5 years after the first implantation. In Spinal Restabilisation Procedures. Edited by Kaech DL and Jinkins JR. Amsterdam: Elsevier; 2002:283-292.

7. Allen MJ, Schoonmaker JE, Bauer TW, et al. Preclinical evaluation of a poly (vinyl alcohol) hydrogel implant as a replacement for the nucleus pulposus. Spine. 2004;29:515-523.

8. Wilke HJ, Heuer F, Neidlinger-Wilke C, et al. Is a collagen scaffold for a tissue engineered nucleus replacement capable of restoring disc height and stability in an animal model? Eur Spine J. 2006;15(Suppl 3): S433-S438.

9. Wilke HJ, Claes L, Schmitt H, et al. A universal spine tester for in vitro experiments with muscle force simulation. Eur Spine J. 1994;3:91-97.

10. Wilke HJ, Neef P, Caimi M, et al. New in vivo measurements of pressures in the intervertebral disc in daily life. Spine. 1999;24:755-762.

11. Wilke HJ, Kavanagh S, Neller S, et al. Effect of a prosthetic disc nucleus on the mobility and disc height of the L4-5 intervertebral disc postnucleotomy. J Neurosurg. 2001;95:208-214.

12. Joshi A, Fussell G, Thomas J, et al. Functional compressive mechanics of a PVA/PVP nucleus pulposus replacement. Biomaterials 2006;27:176-184.

13. Korge A, Nydegger T, Polard JL, et al. A spiral implant as nucleus prosthesis in the lumbar spine. Eur Spine J. 2002;11(Suppl 2): S149-S153.

14. Wilke HJ, Wenger K, Claes L. Testing criteria for spinal implants: recommendations for the standardization of in vitro stability testing of spinal implants. Eur Spine J. 1998;7:148-154.

15. Langrana NA, Parsons JR, Lee CK, et al. Materials and design concepts for an intervertebral disc spacer. I. fiber-reinforced composite design. $J$ Appl Biomater. 1994;5:125-132.

16. Thomas J, Lowman A, Marcolongo M. Novel associated hydrogels for nucleus pulposus replacement. J Biomed Mater Res. 2003;67:13291337.

17. Cotterill PC, Kostuik JP, D'Angelo G, et al. An anatomical comparison of the human and bovine thoracolumbar spine. J Orthop Res. 1986;4:298-303.

18. Wilke HJ, Krischak ST, Wenger KH, et al. Load-displacement properties of the thoracolumbar calf spine: experimental results and comparison to known human data. Eur Spine J. 1997;6:129-137. 